\title{
Structural abnormalities of the brain other than molar tooth sign in Joubert syndrome-related disorders
}

\author{
Efsun U. Şenocak, Kader Karlı Oğuz, Göknur Haliloğlu, Meral Topçu, Ayşenur Cila
}

\begin{abstract}
PURPOSE
We performed a retrospective study in which we investigated malformations other than brainstem and vermian dysgenesis in Joubert syndrome-related disorders (ISRD). We investigated the frequency and type of structural abnormalities that coexist with the molar tooth sign (MTS) in JSRD.

MATERIALS AND METHODS

We searched our archive for the years 2002-2008 in order to find patients with the diagnosis of JSRD. Cranial magnetic resonance imaging studies of 20 patients with the diagnosis of JSRD were reviewed by two neuroradiologists.

RESULTS

In addition to known anomalies including callosal dysgenesis, heterotopia, polymicrogyria, atretic encephalocele, hypomyelination, and nonobstructive dilatation of lateral ventricles; malformations that have not been previously reported were determined, including cerebellar folial disorganization, hippocampal malformation, temporal lob hypoplasia, ambient cistern lipoma, and parenchymal cyst.

CONCLUSION

Structural abnormalities associated with the MTS are not rare, and the additional imaging findings may help explain the neurological presentation in these patients.

Key words: • brain stem • hippocampal formation • cerebellum - magnetic resonance imaging
\end{abstract}

From the Departments of Radiology (E.U.Ş. $\square$ eurger@yahoo. com, K.K.O., A.C.) and Pediatric Neurology (G.H., M.T.), Hacettepe University School of Medicine, Ankara, Turkey.

Received 12 February 2009; revision requested 13 April 2009; revision received 31 May 2009; accepted 2 June 2009.

Published online 26 January 2010 DOI 10.4261/1305-3825.DIR.2673-09.1 oubert et al. first described in 1969 four siblings who had episodes of abnormal breathing and eye movements, ataxia, and mental retardation in association with vermian agenesis and meningoencephalocele in one sibling (1). The name Joubert syndrome (JS) was given several years later when an additional set of patients with similar findings was reported. The neuroradiological hallmark of JS is a complex midbrainhindbrain malformation that creates the molar tooth sign (MTS): (i) thinning of the isthmus with widened interpeduncular fossa; (ii) thickened superior cerebellar peduncles lying perpendicular to the dorsal pons; (iii) hypoplasia of the vermis with enlargement of the fourth ventricle and rostral shift of the fastigium; and (iv) sagittal vermian cleft due to incomplete fusion of the two halves of vermis (2). The absence of a normal vermis leads midline apposition of the cerebellar hemispheres and results in characteristic "batwing" appearance of the fourth ventricle on transverse imaging (3). There is a group of conditions, termed Joubert syndrome-related disorders (JSRDs) with clinical and radiological evidence of JS associated with additional findings and variable involvement of other organs and systems, mainly the eyes and kidneys.

Although hindbrain malformation has been well described in the literature, there appears no detailed study concerning coexisting structural abnormalities in JS, to the best of our knowledge. In this retrospective study, we aimed to investigate the cranial malformations other than MTS in JS and their frequency.

\section{Materials and methods}

Cranial magnetic resonance imaging (MRI) of 20 patients (age range, 18 months-17 years; male/female, 13/7) diagnosed with JS by two experienced pediatric neurologists (GH, MT) were reviewed. Brain MRIs of the patients were performed on a 1.5-T system (Magnetom, Symphony, Siemens Medical Systems, Erlangen, Germany) between 2002 and 2008. All patients had at least the following MR sequences: axial and sagittal T1-weighted (W) spin echo (SE) (TR/TE, 500-600/12 ms; matrix, 192-256; field of view, 230-230 mm), axial and coronal T2W SE (TR/TE, 4000-5000/100 ms; matrix, 192-256; field of view, 230-230 $\mathrm{mm}$ ), and axial fluid-attenuated inversion-recovery (FLAIR) (TR/TE/TI, 8500/98/2150 ms; matrix, 192-256; field of view, 230-230 mm). Two neuroradiologists (KKO, EUS) evaluated MR images in consensus. Following assessment of cerebellar vermis, cerebellar peduncles, and mesencephalon, special attention was paid to evaluation of supratentorial structures and cerebellum. Abnormalities other than MTS were noted.

\section{Results}

All the patients met the primary criteria established by Valente et al. (4). MRI findings are summarized in Table. Seventeen of 20 patients 
Table. Accompanying infra- and supratentorial abnormalities other than molar tooth sign in Joubert syndrome

\begin{tabular}{lc}
\hline $\begin{array}{l}\text { Accompanying } \\
\text { malformations on MRI }\end{array}$ & $\begin{array}{c}\text { Number of patients } \\
\text { (percentage) }\end{array}$ \\
\hline Hippocampal malformation & $16(80 \%)$ \\
Callosal dysgenesis & $16(80 \%)$ \\
Cerebellar folial disorganization & $5(25 \%)$ \\
Temporal lobe hypoplasia & $5(25 \%)$ \\
Nonspecific white matter T2 hyperintensities & $4(20 \%)$ \\
Ventriculomegaly & $2(10 \%)$ \\
Atretic encephalocele & $1(5 \%)$ \\
Ambient cistern lipoma & $1(5 \%)$ \\
Perisylvian polymicrogyri & $1(5 \%)$ \\
Periventricular, subcortical heterotopia & $1(5 \%)$ \\
Hypomyelination & $1(5 \%)$ \\
Bilateral large caudate nuclei & $1(5 \%)$ \\
Parenchymal cyst & $1(5 \%)$ \\
Hyperintense globus pallidus ${ }^{\mathrm{a}}$ & $1(5 \%)$ \\
\hline
\end{tabular}

${ }^{a}$ In a patient with known hepatic fibrosis. supratentorial ventricular dilatation in $2(10 \%)$ and frontal parenchymal cyst in $1(5 \%)$ patient. There were also nonspecific white matter hyperintensities in 4 patients (20\%) and bilateral hyperintense globus pallidus on $\mathrm{T} 1 \mathrm{~W}$ imaging in a patient with known congenital hepatic fibrosis.

\section{Discussion}

The identification of a neuroradiological hallmark of the MTS greatly improved the ease of diagnosing JS. Furthermore, a large number of disorders which display MTS on imaging and variable involvement of other organs and systems-such as the eyes, kidneys, and liver, including occipital (meningo)encephalocele, polydactyly, bilateral ocular colobomas, retinal involvement (in the form of chorioretinal dysplasia, retinal degeneration or Leber congenital amaurosis), cystic kidneys, and autistic behavioral spectrum have been recognized. These disorders were first described in 1999 by Satran et al. (5) as "cerebello-oculorenal syndromes" (CORS), and later expanded by Gleeson et al. (6), who listed eight distinct conditions under the term "Joubert syndrome-related disorders (JSRDs)."

Recently, Valente et al. (4) made a new, simplified nosological classification of JSRDs, and indicated that the main neurological signs of JSRD of hypotonia, ataxia, developmental delay, and oculomotor apraxia, along with a neuroradiologically proven MTS, rep-
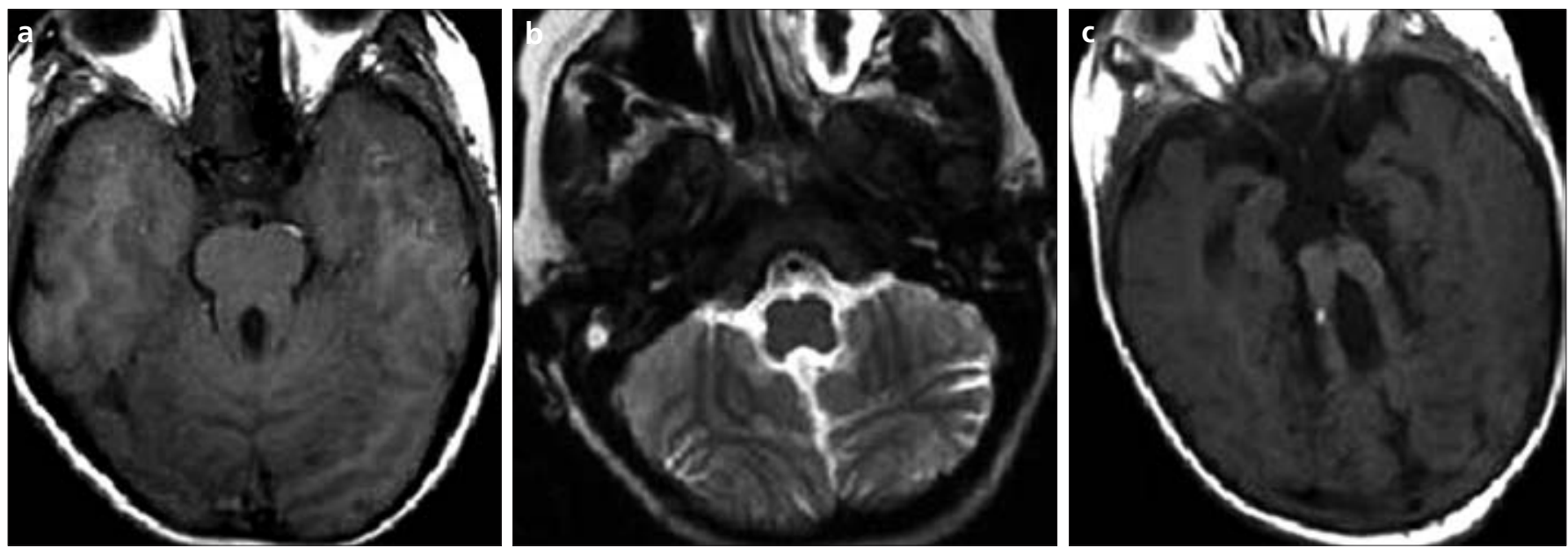

Figure 1. a-c. Axial T1-weighted spin-echo (SE) MR image (TR/TE, 550/15 ms) (a) demonstrates molar tooth appearance. There is vermian hypoplasia with bilateral horizontally-oriented and thickened superior cerebellar peduncles. Axial T2-weighted turbo SE MR image (TR/TE, $3500 / 90 \mathrm{~ms}$ ) (b) shows prominent cerebellar folial disorganization. Axial T1-weighted SE (TR/TE, 550/15 ms) MR image (c) shows molar tooth sign with horizontally-oriented and thickened superior cerebellar peduncles, a deepened interpeduncular fossa, and vermian hypoplasia. There is also a small right ambient cistern lipoma. 

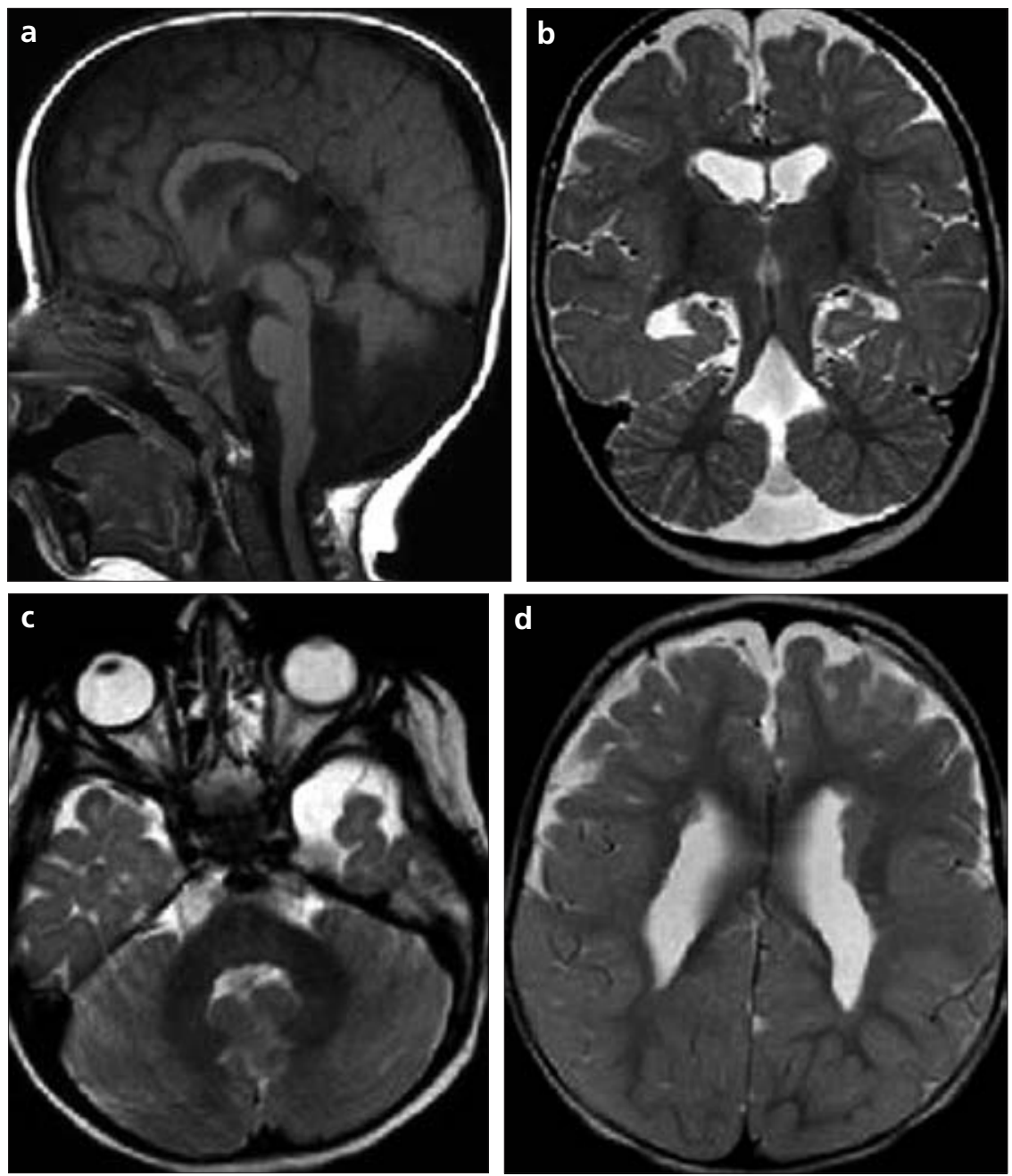

Figure 2. a-d. Sagittal T1-weighted spin-echo (SE) MR image (TR/TE, 530/15 ms) (a) shows a deep interpeduncular cistern. There is inferior vermian hypoplasia and mega cisterna magna. The corpus callosum is short with absence of splenium. Coronal T2-weighted turbo SE MR image (TR/TE, 3700/90 ms) (b) depicts bilateral hippocampal malformation. Both hippocampi have globular shape and are medially located. Temporal horns of the lateral ventricles are enlarged. Axial T2-weighted turbo SE (TR/TE, 3500/90 ms) MR image (c) shows right temporal lobe hypoplasia. Axial T2-weighted turbo SE MR image (TR/TE, 4000/100 ms) (d) demonstrates subependymal periventricular heterotopia and perisylvian polymicrogyria.

resent the unique mandatory features to diagnose JSRD (primary criteria). Six subgroups of JSRDs were then defined on the basis of additional clinical criteria (4).

Central nervous system (CNS) abnormalities other than MTS, such as CC dysgenesis, hydrocephalus, encephalocele, and polymicrogyria, may also be present in all subgroups according to these expanded classifications. Reviewing the literature, we noted that there were few reported cases of supra- and infratentorial abnormalities accompanying brainstem and vermian dysgenesis in JS. Quisling et al. (2) described prominent subarachnoid spaces and ventriculomegaly in a third of the pa- tients in their study. Delayed myelination, fluid collections in posterior fossa resembling Dandy-Walker malformation, hydrocephalus, occipital encephaloceles or meningoceles, agenesis of the CC, polymicrogyria and/or cortical dysplasia, and lissencephaly were described in individual cases. Cerebellar heterotopia has also been noted (7-9). We were unable to find any case of cerebellar folial disorganization, temporal lobe hypoplasia, and ambient cistern lipoma with JS/JSRD in the literature. hippocampal malformations were the most frequent coexistent abnormalities, with a frequency of $80 \%$. There are reported cases of callosal dysgenesis; In our study, callosal dysgenesis and however, to the best of our knowledge, associated hippocampal malformations have not been reported previously. Patients with JS may have epilepsy and detailed examination of these structures can give a clue for seizure etiology. In fact, co-occurrence of CC dysgenesis and hippocampal malformation, most probably due to timing of the insult, has been well described in the literature (10). Other than callosal dysgenesis, hippocampal malformations have been reported with various congenital malformations, including disorders of neuronal migration, non-callosal midline anomalies (including abnormal anterior or hippocampal commissures and interhemispheric cysts and lipomas), and abnormalities of the cerebellum or brainstem (11). Hippocampal malformations including shape and position abnormalities occur at 10 to 30 weeks of gestation (12), whereas CC develops between 8 and 20 weeks of gestation (7). At 5-10 weeks of gestation, the prechordal mesoderm induces face and forebrain, the rhombencephalon gives rise to the cerebellar hemispheres and vermis, and the myelencephalon gives rise to the medulla and pons. Insult during this period may result in MTS of JS, cerebellar hypo-/dysplasia, and facial anomalies. Since formation of the germinal matrix occurs at about 7 weeks' gestational age and neuronal migration from germinal matrix to cortex and subsequent layering take place between weeks 6-7 through 24-26, one can expect accompanying migrational abnormalities in JS. An insult at 3-4 weeks of gestation, when chordal mesoderm induces the neural plate, followed by closure of the neural plate and then the neural tube, may result in cephalocele or myelomeningocele (13). It is concluded that genetic and environmental factors during this developmental period (most probably between 3 weeks and 26 weeks of gestation) may cause embryological abnormalities, affecting both supra- and infratentorial structures (7). Today, JSRDs are included in the rapidly expanding group of disorders called ciliopathies, because all six gene products implicated in JSRD (NPHP1, AHI1, CEP290, RPGRIP1L, TMEM67, and ARL13B) function in the primary cilium/basal body organelle (14). Recently Gorden et al. (14) identified lossof-function mutations in CC2D2A in JSRD patients with and without retinal, kidney, and liver disease. 
Conventional cranial MRI features of JSRD are well known today. In a functional MR imaging study, Parisi et al. (15) demonstrated a striking bilateral activation of the sensorimotor and cerebellar cortex in a patient with JS, in contrast to the typical highly lateralized activation seen in control subjects. Using diffusion tensor imaging and tractography, Lee et al. (16) showed thickened superior cerebellar peduncles in three patients, and Widjaja et al. (17) found horizontally oriented superior cerebellar peduncles that failed to decussate and laterally located deep cerebellar nuclei in two patients with JS. Poretti et al. (18) showed the absence of decussation of the superior cerebellar peduncles and corticospinal tract and the more lateral localization of the deep cerebellar nuclei.

Reported neuropathologic abnormalities are limited to hindbrain structures. These include distorted bandlike structure of the dentate nuclei and grouping of its neurons into clusters or islands (many small islands of heterotopic gray matter scattered throughout the white matter), and abnormalities in the medulla, including dysplasia of the nuclei and tracts $(19,20)$. Extensive brainstem malformation may explain the oculomotor apraxia and hyperpnea (21).

The recurrence risk is $25 \%$ (22), and an antenatal investigation of JS by ultrasound and/or MRI must be performed in cases with a sibling with JS (23).

In conclusion, revealing that a wide range of CNS abnormalities may coexist with characteristic MTS, this study supports heterogeneity in this complex syndrome derived from genetic studies and phenotypic observations. Further embryological, molecular, and genetic studies are needed to understand the underlying pathologies that affect various anatomic structures in JS. More detailed analysis with high-resolution imaging is needed for better definition of overlapping and distinct neuroanatomic features. In clinical practice, radiologists should scrutinize abnormalities other than molar tooth malformation, since in some situations it is these accompanying findings that may explain the symptoms of patients.

\section{References}

1. Joubert M, Eisenring JJ, Robb JP Andermann F. Familial agenesis of the cerebellar vermis. A syndrome of episodic hyperpnea, abnormal eye movements, ataxia and retardation. Neurology 1969; 19:813-825.

2. Quisling RG, Barkovich AJ, Maria BL Magnetic resonance imaging features and classification of central nervous system malformations in Joubert syndrome. J Child Neurol 1999; 14:628-635.

3. McGraw P. The molar tooth sign. Radiology 2003; 229:671-672.

4. Valente EM, Brancati F, Dallapiccola B. Genotypes and phenotypes of Joubert syndrome and related disorders. Eur J Med Genet 2008; 51:1-23.

5. Satran D, Pierpont ME, Dobyns WB. Cerebello-oculo-renal syndromes including Arima, Senior-Löken, and COACH syndromes: more than just variants of Joubert syndrome. Am J Med Genet 1999; 86:459_ 469.

6. Gleeson JG, Keeler LC, Parisi MA, et al. Molar tooth sign of the midbrain-hindbrain junction: occurrence in multiple distinct syndromes. Am J Med Genet 2004; 125:125-134.

7. Zamponi N, Rossi B, Messori A, Polonara G, Regnicolo L, Cardinali C. Joubert syndrome with associated corpus callosum agenesis. Eur J Paediatr Neurol 2002; 6:6366.

8. Parisi MA, Doherty D, Chance PF Glass IA. Joubert syndrome (and related disorders) (OMIM 213300). Eur J Hum Genet 2007; 15:511-521.

9. Ozyurek H, Kose G. Joubert syndrome associated with lissencephaly. Indian Pediatr 2005; 42:494-495.

10. Hetts SW, Sherr EH, Chao S, Gobuty S, Barkovich AJ. Anomalies of the corpus callosum: an MR analysis of the phenotypic spectrum of associated malformations. AJR Am J Roentgenol 2006; 187:1343-1348.
11. Sato N, Hatakeyama S, Shimizu N, Hikima A, Aoki J, Endo K. MR evaluation of the hippocampus in patients with congenital malformations of the brain. AJNR Am J Neuroradiol 2001; 22:389-393.

12. Baulac M, De Grissac N, Hasboun D, A et al. Hippocampal developmental changes in patients with partial epilepsy: magnetic resonance imaging and clinical aspects. Ann Neurol 1998; 44:223-233.

13. Osborn AG, Blazer S, Salzman KL. Diagnostic imaging: brain, 1st ed. Salt Lake City: Amirsys, 2004; 10-15.

14. Gorden NT, Arts HH, Parisi MA, et al. CC2D2A is mutated in Joubert syndrome and interacts with the ciliopathy-associated basal body protein CEP290. Am J Hum Genet 2008; 83:559-571.

15. Parisi MA, Pinter JD, Glass IA, et al. Cerebral and cerebellar motor activation abnormalities in a subject with Joubert syndrome: functional magnetic resonance imaging (MRI) study. J Child Neurol 2004; 19:214-218.

16. Lee SK, Kim DI, Kim J, et al. Diffusion-tensor MR imaging and fiber tractography: a new method of describing aberrant fiber connections in developmental CNS anomalies. Radiographics 2005; 25:53-68.

17. Widjaja E, Blaser S, Raybaud C. Diffusion tensor imaging of midline posterior fossa malformations. Pediatr Radiol 2006; 36:510-517.

18. Poretti A, Boltshauser E, Loenneker T, et al. Diffusion tensor imaging in Joubert syndrome. AJNR Am J Neuroradiol 2007; 28:1929-1933.

19. Sztriha L, Al-Gazali LI, Aithala GR, Nork M. Joubert's syndrome: new cases and review of clinicopathologic correlation. Pediatr Neurol 1999; 20:274-281.

20. Subramanian S, Hari S, Santosh Kumar S. Clinics in diagnostic imaging (118). Singapore Med J 2007; 48:869-872.

21. Yachnis AT, Rorke LB. Neuropathology of Joubert syndrome. J Child Neurol 1999; 14:655-659.

22. Alorainy IA, Sabir S, Seidahmed MZ, Farooqu HA, Salih MA. Brain stem and cerebellar findings in Joubert syndrome. J Comput Assist Tomogr 2006; 30:116-121.

23. Doherty D, Glass IA, Siebert JR, et al. Prenatal diagnosis in pregnancies at risk for Joubert syndrome by ultrasound and MRI. Prenat Diagn 2005; 25:442-447. 